\title{
BMJ Open Incidence of HIV and HCV in people who inject drugs: a systematic and meta- analysis review protocol
}

\author{
Asaad Sharhani, ${ }^{1}$ Zahra Jorjoran Shushtari, ${ }^{2}$ Azam Rahmani, ${ }^{3}$ Bahram Armoon (D) , \\ Mehdi Noroozi, ${ }^{2}$ Elaheh Ahounbar, ${ }^{5}$ Salah Eddin Karimi (i) , ${ }^{6}$ Peter Higgs ${ }^{7,8}$
}

To cite: Sharhani A, Jorjoran Shushtari Z, Rahmani A, et al. Incidence of HIV and HCV in people who inject drugs: a systematic and meta-analysis review protocol. BMJ Open 2021;11:e041482. doi:10.1136/ bmjopen-2020-041482

- Prepublication history for this paper is available online. To view these files, please visit the journal online (http://dx.doi. org/10.1136/bmjopen-2020041482).

Received 10 June 2020 Revised 01 December 2020 Accepted 12 December 2020

Check for updates

(c) Author(s) (or their employer(s)) 2021. Re-use permitted under CC BY-NC. No commercial re-use. See rights and permissions. Published by BMJ.

For numbered affiliations see end of article.

Correspondence to Dr Salah Eddin Karimi; salahkarimi2009@gmail.com

\section{ABSTRACT}

Introduction HIV and hepatitis $\mathrm{C}$ virus (HCV) are major health concerns globally. This systematic review and metaanalysis protocol study aims to estimate the incidence of HIV and HCV among people who inject drugs (PWIDs) by reviewing studies that have applied mathematical modelling. The primary purpose of this systematic review is to identify and review mathematical modelling studies of HIV and HCV incidence in PWIDs.

Methods and analysis cohort, cross-sectional and clinical trial studies conducted to estimate the incidence of HIV and HCV based on mathematical models or have evaluated the effectiveness of mathematical models will be considered for inclusion in the review. A comprehensive search applying a Cochrane approach will be used to identify relevant primary studies, published between January 2000 and July 2020, and indexed in PubMed, EMBASE, Opengrey, WOS, SCOPUS and Cochrane Library with no restriction on language. This protocol was prepared according to the Preferred Reporting Items for Systematic Review and Meta-Analysis Protocols (PRISMA-P). Study selection and data extraction will be performed by two independent reviewers. Assessment of risk of bias will be implemented using forms of the Critical Appraisal Skills Programme. Publication bias will be assessed by funnel plots, Begg's and Egger's tests. A meta-analysis will be conducted to answer the first research question, 'What is the incidence of HIV and HCV when applying mathematical model in PWID?'. Clinical heterogeneity will be assessed by looking at the characteristics of participants, method of diagnosis and case definitions in the included primary studies. In addition, subgroup analyses will be conducted for population and secondary outcomes.

Ethics and dissemination There are no ethical issues related to this study. The findings will be published in peerreviewed scientific journals and presented at international and national conferences.

PROSPERO registration number CRD42019126476.

\section{INTRODUCTION}

Despite significant progress in the treatment of chronic diseases over the past decade, HIV remains a major health concern globally with 36.7 million people infected-it is predicted to be the third main cause of death by $2030{ }^{1}$ Although epidemiological evidence indicates a decreasing trend in many developed

\section{Strengths and limitations of this study}

The present systematic review is the first to examine models for the incidence of HIV and hepatitis $C$ virus (HCV) in injecting drug users by searching various databases.

- To minimise potential bias, each process of initial screening, data extraction and quality evaluation will be performed by two independent reviewers.

- The study is not limited by specific languages and therefore is not exposed to language bias.

- This review only includes studies using mathematical models, therefore, it may ignore some studies working on HIV and HCV incidence in people who inject drugs.

countries during the last few years, HIV prevalence is increasing in developing countries. ${ }^{2}$

The UNAIDS global strategy for 2016-2021 focuses on the application of effective interventions to reduce the burden of HIV among key populations. ${ }^{3}$ One of the main at-risk populations for HIV infection are people who inject drugs (PWIDs). According to the literature, $13.3 \%$ of injecting drug users are infected by HIV, with a remarkable rate of $28.8 \%$ in South East Asia and 23\% in Eastern Europe. ${ }^{4}$

The sharing of previously used injection equipment potentially exposes PWID to HIV infection, ${ }^{5}$ and is one of the main routes for HCV transmission. ${ }^{4}$ Numerous harmreducing interventions have been implemented to limit injection equipment sharing among PWID,${ }^{6-11}$ the most common of which are needle syringe distribution programmes. ${ }^{12}$ The main purpose of which is to reduce opportunities for needle and syringe sharing and to provide education about safer drug use, sexual health and referral for social supports if necessary. ${ }^{1}$

Although harm reduction programmes have been successful in controlling HIV among PWIDs, universal implementation 
has remained challenging. ${ }^{13-16}$ Evaluating and demonstrating the effectiveness of harm reduction programmes among PWID is required if we are to develop the most effective strategies for preventing HIV and HCV infection. ${ }^{17}$ Studies focused on evaluating the effectiveness of harm reduction programmes especially needle syringe programmes (NSP) are often conducted using costeffectiveness principals or with modelling approaches. ${ }^{18}$

Modelling approaches play an important role in evaluating the economic and demographic effects of disease epidemiology, estimating the effectiveness of interventions, and providing precise information to policy makers so decisions can be made about how best to deal with the challenges for controlling infectious diseases. ${ }^{19}{ }^{20} \mathrm{~A}$ recent review of studies showed that "NSP was effective in reducing HIV transmission among PWID, while there were mixed results regarding a reduction of HCV infection, also Full harm reduction interventions provided at the structural level and in multi-component programmes, as well as high level of coverage, were more beneficial". ${ }^{18}$

Using the evidence of an updated systematic review could assist policy makers and health planners to identify the most appropriate health policies and better use resources to plan prevention interventions targeting PWID. This systematic review of HIV and HCV incidence in PWID may clarify underlying causal factors affecting transmission.

\section{Objective}

The objective is to estimate the incidence of HIV and HCV among PWIDs.

\section{Review question(s)}

1. What is the incidence of HIV and HCV when applying a mathematical model in PWID?

2. Heterogeneity assessment and finding potential causes.

\section{METHODS}

This is a protocol that was registered in the PROSPERO (registration number: CRD42019126476) to complete a systematic review using the search strategy to identify relevant primary studies that have been published between January 2000 and July 2020, and indexed in electronic databases including PubMed, EMBASE, Opengrey, WOS, SCOPUS and Cochrane Library. The reference lists of relevant primary studies will also be searched manually to include all relevant studies. A sensitive search strategy will be used to retrieve relevant studies. Both free-text terms and terms at keyword databases such as Medical Subject Headings (MeSH), Emtree and DeCS will be used to define the keywords; for example, 'viral hepatitis C', 'hepatitis virus C', 'HCV'. Meanwhile, common terms in the field of harm reduction and studies regarding PWID will be used in our search strategy. Various forms of words, synonyms, plural words, abbreviations, truncation, and acronyms for 'distribution of syringe', 'harm reduction', 'effectiveness', 'injecting drug' and so on will also be used in varying combinations. The search strategy that will be
Table 1 PubMed search strategy for studies published between January 2000 and July 2020

\begin{tabular}{ll}
\hline Search terms & Search \\
\hline (HIV [Mesh] OR 'HIV infection' OR AIDS OR & $\# 1$ \\
'Human immunodeficiency virus' OR 'Acquired & \\
immune deficiency virus' OR AIDS OR 'Acquired & \\
immunodeficiency syndrome' OR 'Acquired & \\
immunologic deficiency syndrome' OR 'Acquired & \\
immune deficiency syndrome*' [tiab)) &
\end{tabular}

('Viral hepatitis C' OR 'Hepatitis C' OR HCVOR 'Hepatitis C virus' OR "Hepatitis C"(Mesh Terms] (tiab))

('Mathematical models' OR Models OR \#2 Theoretical [Mesh] OR 'Evaluated effectiveness model' [tiab))

('People who inject drugs' OR 'injecting drug \#4 us*' OR 'Syringe exchange Programs' OR 'Needle Sharing' [Mesh] OR 'Drug

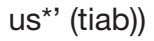

\#1 AND \#2 AND \#3 AND \#4 AND \#5

used for PubMed is reported in table 1 . We will modify our search strategy to suit each database. We will update the search 6 months ahead of publishing the systematic review paper. All results will be managed by EndNote software. Duplicate records will be recognised and removed.

\section{Eligibility criteria (inclusion and exclusion criteria)}

Any studies completed with PWID in all settings will be considered for review. Therefore, Drop-in Centres (DIC) and drug market neighbourhoods will both be considered.

Studies that were conducted to estimate the incidence of HIV and HCV based on mathematical models or have evaluated the effectiveness of mathematical models will be considered for the review.

This systematic review will include all cohort, clinical trials and cross-sectional studies. As the main focus of this study will be on estimating HIV and HCV incidence through mathematical models, only studies that have applied mathematical models in order to evaluate HIV and HCV incidence will be enrolled in the review.

Inclusion criteria based on PICOs (Cochrane standard) are as follows:

P (Participants or population): Studies with PWID as the study population. Any studies that have been completed with PWID across all settings will be enrolled for review. Therefore, the DIC and drug market neighbourhoods will also be considered.

I (Intervention) and C (comparison) are not relevant to this protocol study.

O (Outcome): Estimates for the incidence of HIV and HCV among PWID based on mathematical models; only studies that were conducted to estimate the incidence of HIV and HCV based on mathematical models will be considered. 
S: Cohort studies and studies that evaluated the effectiveness of mathematical models in estimating the incidence of HIV and HCV in PWID. This systematic review will include all cohort, clinical trials and cross-sectional studies. The main focus of this study will be estimating HIV and HCV incidence through mathematical models. Only studies that apply mathematical models in order to evaluate HIV and $\mathrm{HCV}$ incidence will be enrolled in the review.

\section{Exclusion criteria}

Studies with the population(s) other than PWID, qualitative studies and studies with a secondary analysis will be excluded from the review. Also, studies that estimate HIV and HCV incidence directly or through laboratory tests and self-report data will be excluded from the study.

\section{Study selection}

Initially, at least two authors will evaluate studies according to title and abstract and the chosen full texts that comply with the inclusion criteria will be entered for full-text review. Questions will be designed for each section based on inclusion criteria. Finally, articles that meet eligibility for the study questions and inclusion criteria will be entered for further evaluation and data extraction.

Full texts of studies identified by both authors as eligible will be sourced and the full texts of studies recognised as eligible by one author will be evaluated based on the inclusion criteria. If further information is needed, we will contact their first or corresponding authors to collect any required information. A third author will be consulted to make any final decisions regarding inclusion in the review. Reasons for exclusion will be recorded.

\section{Assessing the quality of articles}

Two reviewers will independently appraise the study quality and risk of bias using adapted forms of the Critical Appraisal Skills Programme (CASP) ${ }^{21}$ for cross-sectional and cohort studies, and consort Critical Appraisal Skills for randomised trial studies will be used. Quality scores will be calculated from the individual items in the checklists. The mean quality score will be calculated as the quality sum score of each article divided by the number of items in the critical appraisal forms.

\section{Extraction and data management}

Two of the study authors will separately extract data in accordance with the designed form using the information provided in articles. Data from papers reporting the results of one study will be combined together into one single study. Articles in languages other than English or Persian will be translated into Persian. The process outlined above will be administered on any of these papers.

Data extraction will be done by collecting the results of relevant studies and recording these in an Excel spreadsheet. The data collection form will include authors' name, type of study, place of the study, year of publication, study population, sample size and applied methodology in the study.

\section{Method of analysis and assessment of heterogeneity}

After completing the data extraction phase, the results of the included papers will be categorised and summarised in descriptive tables. Publication bias will be assessed by funnel plots, Begg's and Egger's tests. We are planning to conduct a meta-analysis to answer the second research question. Clinical heterogeneity will be assessed by looking at the characteristics of participants, method of diagnosis and case definitions in the included primary studies. Also, we will use the $\chi^{2}$ test and $\mathrm{I}^{2}$ statistics to choose between fixed effects and random effects metaanalysis. In addition, subgroup analyses will be conducted for the study population and the secondary outcomes. All meta-analyses will be performed using Stata V.12 software.

In case the level of interval confidence for evaluating effectiveness overlaps among studies, or $\mathrm{I}^{2}$ statistics is higher than $40 \%$ or $p$ value in $\chi^{2}$ test for two heterogeneities is less than 0.1 , then there is heterogeneity between the studies, and it is not possible to conduct fixed method meta-analysis, then random effects used to meta-analysis if the $\mathrm{I}^{2}$ value is $>40 \%$.

In the meta-analyses, we will pay attention to the status of incidence based on geographical areas, and in case of differences in incidence in specific geographical areas, geographic area sensitivity analyses will be performed.

\section{Patient and public involvement}

Patients and the public were not involved with the development of this protocol. The results will be published in open-access, peer-reviewed publications.

\section{DISCUSSION}

This systematic review will provide incidence estimates for HIV and HCV in PWID using mathematical models. The synthesis of review findings will assess study limitations as well as any limitations in our own review methodology. Once a large volume of studies has been identified from the first search, we will use a multiple reviewer team to minimise the risk of bias. A team of authors is beneficial in reducing the time needed to complete the study. The findings of this review study may also be compared with findings from other studies on this issue. Finally, in the discussion, key findings, study limitations, implications and recommendations for future research, and practical/clinical considerations for specialists will be presented.

This review only includes studies using mathematical models therefore, it may exclude other important studies working on HIV and HCV incidence in PWIDs.

In summary, this review study will produce robust data on the incidence of HIV and HCV in PWIDs. It is expected that the findings of this review will be of interest to infectious disease physicians, psychiatrists, drug and alcohol specialists, harm reduction professionals, policy makers and others working with PWID. 


\section{ETHICS AND DISSEMINATION}

This study will synthesise the relevant primary studies on the incidence of HIV and HCV in PWIDs. Results of this review study will be disseminated in peer-reviewed scientific journals and may be presented at international and national conferences to help researchers, harm reduction professionals, healthcare workers and policy makers in the development of future research studies, effective interventions and health planning to prevent HIV and HCV among this at-risk population. Also, this review study will provide important detailed information about study design, ethical and operational challenges, and valid findings (controlled for biases) for future investigations and interventions.

This is an open access article distributed in accordance with the Creative Commons Attribution Non-Commercial (CC BY-NC 4.0) license, which permits others to distribute, remix, adapt, build on this work non-commercially and license their derivative works on different terms, provided the original work is properly cited, appropriate credit is given, any changes made indicated and the use is noncommercial. See: http://creativecommons.org/licenses/ by-nc $/ 4.0 /$.

\section{IMPLICATIONS OF KEY FINDINGS}

Results of this review will help researchers, healthcare workers and policy makers in developing research studies, effective interventions and health protocols or guidelines to promote best outcomes for this at-risk population. Also, this review will provide important detailed information about study design, ethical and operational challenges, and valid findings (controlled for biases) for future investigations and interventions.

\section{Author affiliations}

${ }^{1}$ Department of Epidemiology and Biostatistics, Public Health Faculty, Ahvaz Jundishapur University of Medical Sciences, Ahvaz, Iran

${ }^{2}$ Social Determinants of Health Research Center, University of Social Welfare and Rehabilitation Sciences, Tehran, Iran

${ }^{3}$ Nursing and Midwifery Care Research Center, School of Nursing and Midwifery, Tehran University of Medical Sciences, Tehran, Iran

${ }^{4}$ Social Determinants of Health Research Center, Saveh University of Medical Sciences, Saveh, Iran

${ }^{5}$ Substance Abuse and Dependence Research Center, University of Social Welfare and Rehabilitation Sciences, Tehran, Iran

${ }^{6}$ Social Determinants of Health Research Center, Health Management and Safety Promotion Research Institute, Tabriz University of Medical Sciences, Tabriz, Iran ${ }^{7}$ Department of Public Health, La Trobe University, Plenty Rd \& Kingsbury Dr, Bundoora, VIC 3086, La Trobe University, Melbourne, Victoria, Australia ${ }^{8}$ Behaviours and Health Risks Program, Burnet Institute, 85 Commercial Road, Melbourne, VIC 3004, Burnet Institute, Melbourne, Victoria, Australia

\section{Twitter Peter Higgs @higgspg}

Acknowledgements The authors would like to thank all those who have contributed to the preparation of this protocol.

Contributors ZJS, AR, AS and SEK drafted the protocol. PH, MN, BA, AR and EA commented on and suggested revisions to the protocol. All authors approved the final revision of the protocol.

Funding The authors have not declared a specific grant for this research from any funding agency in the public, commercial or not-for-profit sectors.

Competing interests None declared.
Patient and public involvement Patients and/or the public were not involved in the design, or conduct, or reporting or dissemination plans of this research.

Patient consent for publication Not required.

Provenance and peer review Not commissioned; externally peer reviewed.

Open access This is an open access article distributed in accordance with the Creative Commons Attribution Non Commercial (CC BY-NC 4.0) license, which permits others to distribute, remix, adapt, build upon this work non-commercially, and license their derivative works on different terms, provided the original work is properly cited, appropriate credit is given, any changes made indicated, and the use is non-commercial. See: http://creativecommons.org/licenses/by-nc/4.0/.

ORCID iDs

Bahram Armoon http://orcid.org/0000-0001-5467-9889

Salah Eddin Karimi http://orcid.org/0000-0002-1542-0214

\section{REFERENCES}

1 Sheet F. Joint United nations programme on HIVIAIDS (UNAIDS). Global Statistics, 2014.

2 Baral S, Beyrer C, Muessig K, et al. Burden of HIV among female sex workers in low-income and middle-income countries: a systematic review and meta-analysis. Lancet Infect Dis 2012;12:538-49.

3 UNAIDS. UNAIDS 2016-2021 strategy. Available: http://www.unaids. org/en/aboutunaids/unaidsstrategygoalsby2015 [Accessed 22 Feb 2016].

4 Degenhardt L, Charlson F, Stanaway J, et al. Estimating the burden of disease attributable to injecting drug use as a risk factor for HIV, hepatitis $\mathrm{C}$, and hepatitis $\mathrm{B}$ : findings from the global burden of disease study 2013. Lancet Infect Dis 2016;16:1385-98.

5 Stimson GV. The global diffusion of injecting drug use: implications for human immunodeficiency virus infection. Bull Narc 1993;45:3-17.

6 Garfein RS, Golub ET, Greenberg AE, et al. A peer-education intervention to reduce injection risk behaviors for HIV and hepatitis $C$ virus infection in young injection drug users. AIDS 2007;21:1923-32.

7 Hunt N, Ashton M, Lenton S, et al. A review of the evidence-base for harm reduction approaches to drug use. London: Forward Thinking on Drugs, 2003.

8 Go VF, Frangakis C, Minh NL, et al. Efficacy of a multi-level intervention to reduce injecting and sexual risk behaviors among HIVinfected people who inject drugs in Vietnam: a four-arm randomized controlled trial. PLoS One 2015;10:e0125909.

9 Bruneau J, Lamothe F, Franco E, et al. High rates of HIV infection among injection drug users participating in needle exchange programs in Montreal: results of a cohort study. Am J Epidemiol 1997;146:994-1002.

10 Aceijas C, Hickman M, Donoghoe MC, et al. Access and coverage of needle and syringe programmes (NSP) in central and eastern Europe and central Asia. Addiction 2007;102:1244-50.

11 Bluthenthal RN, Ridgeway G, Schell T, et al. Examination of the association between syringe exchange program (SEP) dispensation policy and SEP client-level syringe coverage among injection drug users. Addiction 2007;102:638-46.

12 Rhodes T, Hedrich D. Harm reduction: evidence impacts and challenges: office for official publications of the European communities, 2010.

13 Magada ES. An investigation into the influence of socio-cultural factors on HIV prevention strategies: a case study of HIV serodiscordant couples in Harare-Zimbabwe, 2014.

14 Bio-behavioral survey on drug users and sexual partners in Iran 2011.

15 National AIDS Committee Secretariat, Ministry of Health and Medical Education. Islamic Republic of Iran AIDS progress report: on monitoring of the United nations General assembly special session on HIV and AIDS. Tehran, Iran, 2015.

16 Stöver H, Hariga F. Prison-based needle and syringe programmes (PNSP)-still highly controversial after all these years. Drugs: Educat Prevent Policy 2016;23:103-12.

17 Wilson DP, Donald B, Shattock AJ, et al. The cost-effectiveness of harm reduction. Int J Drug Policy 2015;26:S5-11.

18 Fernandes RM, Cary M, Duarte G, et al. Effectiveness of needle and syringe Programmes in people who inject drugs - An overview of systematic reviews. BMC Public Health 2017;17:309.

19 Ball AL, Rana S, Dehne KL. HIV prevention among injecting drug users: responses in developing and transitional countries. Public Health Rep 1998;113:170.

20 Bastos FI, Strathdee SA. Evaluating effectiveness of syringe exchange programmes: current issues and future prospects. Soc Sci Med 2000;51:1771-82.

21 CASP. Available: http://www.casp-uk.net/\#!casp-tools-checklists/ c18f8 